\section{Diode laser assisted excision of a gingival pyogenic granuloma: A case report} Eleftherios Anagnostou, Athanasios Poulopoulos, ${ }^{1}$ Prashanth Panta, ${ }^{2}$ Shankargouda Patil ${ }^{3}$

${ }^{1}$ Department of Oral

Medicine/Pathology, School of Dentistry, Aristotle University of Thessaloniki, Greece; ${ }^{2}$ Department of Oral Medicine and Radiology, MNR Dental College and Hospital, Sangareddy, Telangana, India; ${ }^{3}$ Department of Maxillofacial Surgery and Diagnostic Sciences, Division of Oral Pathology, College of Dentistry, Jazan University, Jazan, Saudi Arabia

\begin{abstract}
Pyogenic granuloma is a non-neoplastic, exuberant, reactive lesion seen in response to local irritation or trauma caused by dental calculus, bacterial plaque, caries and restorations, with a strong predilection for the gingiva. It is among the frequently encountered oral lesions, occurring at a challenging oral site, the gingiva. Herein, we report a 71-year-old medically compromised Caucasian female who presented with a relatively large lobulated pyogenic granuloma on the buccal gingiva of the maxillary molar-premolar region. Total surgical excision was performed with an $840 \mathrm{~nm}$ diode laser followed by the placement of hyaluronate gel and relevant periodontal dressing. Two weeks after surgical excision, complete healing was observed, but patient denied replacement of dental restoration. After 4 months of follow-up, an overall reduction of associated teeth mobility was also observed, with a minor recurrence in gingival inflammation. The objective of this report is to briefly review clinical, radiographic and histological findings of pyogenic granuloma along with a detailed discussion on its management through a diode laser.
\end{abstract}

\section{Introduction}

Pyogenic granuloma (PG) is a common, acquired, benign vascular reactive hyperplasia of the skin or mucosa. ${ }^{1-3}$ The most frequent intra-oral site for $\mathrm{PG}$ is marginal gingiva, but lesions also occur on palate, buccal mucosa, tongue, and lips. Extraorally, it has been reported on the skin of face, neck, upper and lower extremities and mucous membrane of nose and eyelids. ${ }^{2,4}$ Clinically, an oral PG appears as a tumor-like, painless, exophytic mass with erythematous papules that tend to bleed easily, and sizes ranging from a few millimeters to a few centimeters have been reported. 3,4 The color of the lesion may vary according to the level of vascularity. ${ }^{4} \mathrm{PG}$ can develop as a result of chronic, local, low-grade trauma or irritation and due to hormonal factors. ${ }^{3,4}$ Many treatment modalities were applied for oral PG. Conventional surgical excision can be used but some complications like intra-operative bleeding and postoperative infection may occur, which can affect the dynamics of healing process. Local irritants and other traumatic factors must be diminished in order to avoid recurrence. ${ }^{3}$ During the past years other treatment modalities like cryosurgery, and laser surgery are being implemented. ${ }^{3,4}$

Laser technology is being widely used in dentistry. Their ability to perform deep and precise incisions, better hemostasis and less invasive procedures with less discomfort to patients have made them a preferred treatment option for several soft tissue lesions. ${ }^{5}$ The advantages of laser surgery compared with conventional surgical methods includes the maintenance of sterile conditions, good estimation of cutting depth, reduction in the number of operative instruments, often no need for suturing or bandages, pain reduction both intra- and postoperatively, promotion of wound healing, less scars, staff and time. ${ }^{6}$ A number of lasers have been successfully used to treat PG, such as neodymium doped yttrium aluminium garnet (Nd:YAG), carbon dioxide laser, erbium-doped yttrium aluminum garnet (Er:YAG), and the diode laser. ${ }^{3}$ In this report we present the successful application of diode laser in the context of a large pyogenic granuloma.

\section{Case Report}

A 71-year-old Caucasian female with a medical history of diabetes mellitus (type II) and hypertension under pharmaceutical treatment (apixaban and nifedipine) presented with a painless gingival growth that appeared 5 months ago. Intraoral examination revealed a solid and lobulated mass with a diameter more than $2 \mathrm{~cm}$, in the marginal and buccal gingiva of the maxillary right molar-premolar region. It was pink in color, bleeded spontaneously, and showed an ulcerated region especially in the molar region (Figure 1). The relevant teeth (\#14 and \#16) had Type II mobility and were covered by metal-ceramic crowns with
Correspondence: Prashanth Panta, Department of Oral Medicine and Radiology, MNR Dental College and Hospital, Narsapur Road, Sangareddy 502294, Telangana, India. E-mail: maithreya.prashanth@gmail.com

Key words: Pyogenic granuloma; Gingiva; Laser surgery; Diode laser.

Contributions: DA, IL, EA, AP: diagnosis, clinical management, follow-up and drafting of preliminary manuscript; PP, SP: contributions to design of work, critical revision for important intellectual content. All of the authors (DA, IL, EA, AP, PP, SP): final revision and approval of the version to be published.

Conflict of interest: the authors declare no potential conflict of interest.

Funding: none.

Ethical statement: the informed consent was obtained from the patient.

Conference presentation: the case was presented as poster clinical presentation at the Europerio 9, June 20-23, 2018.

Received for publication: 16 June 2019.

Revision received: 12 August 2019.

Accepted for publication: 23 August 2019.

This work is licensed under a Creative Commons Attribution NonCommercial 4.0 License (CC BY-NC 4.0).

(C) Copyright: the Author(s), 2019

Licensee PAGEPress, Italy

Clinics and Practice 2019; 9:1179

doi:10.4081/cp.2019.1179

irregular margins. A digital periapical radiograph (RVG 6200 Care-stream, USA) revealed no osseous involvement (Figure 2).

Due to the excessive bleeding tendency observed during clinical examination, an $840 \mathrm{~nm}$ diode laser was chosen as a minimally invasive procedure to excise the lesion. After infiltration of local anesthesia, diode laser (Epic X, BIOLASE, USA) at $840 \mathrm{~nm}$ wavelength in continuous wave mode with a power output of 4.75 watt and a $0.4-\mathrm{mm}$ diameter fiber optic was set for total excision of lesion (Figure 3 ). The tip was directed, moving around the base of the lesion. After excision, hyaluronate and sucralfate oral gel (Hy-AL oral gel, Intermed SA, Greece) and overlying periodontal dressing (Septo-pack, Septodont, France) was placed and left in situ (Figure 4). Histological examination with hematoxylin and eosin staining revealed high vascular proliferation (Figure 5A) and 
inflammatory granulomatous tissue (Figure $5 B)$ with infiltration of lymphocytes, plasma cells and neutrophils along with areas of fibrous connective tissue. The lesion was covered by ulcerated stratified squamous epithelium without atypia. These features confirmed the diagnosis of PG. After total

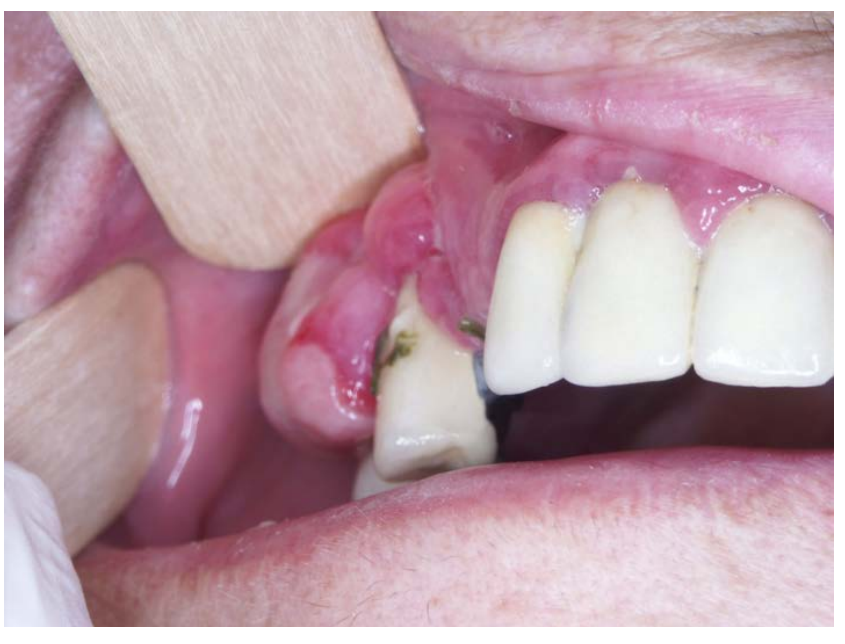

Figure 1. A solid and lobulated pinkish mass with a diameter more than $2 \mathrm{~cm}$ is associated with local ulceration on marginal, buccal gingiva of maxillary right molar. The relevant teeth (\#14 and \#16) are covered by metal-ceramic crowns for partial denture with irregular margins. Circumferential dental calculus is visible around the metal crown of the premolar

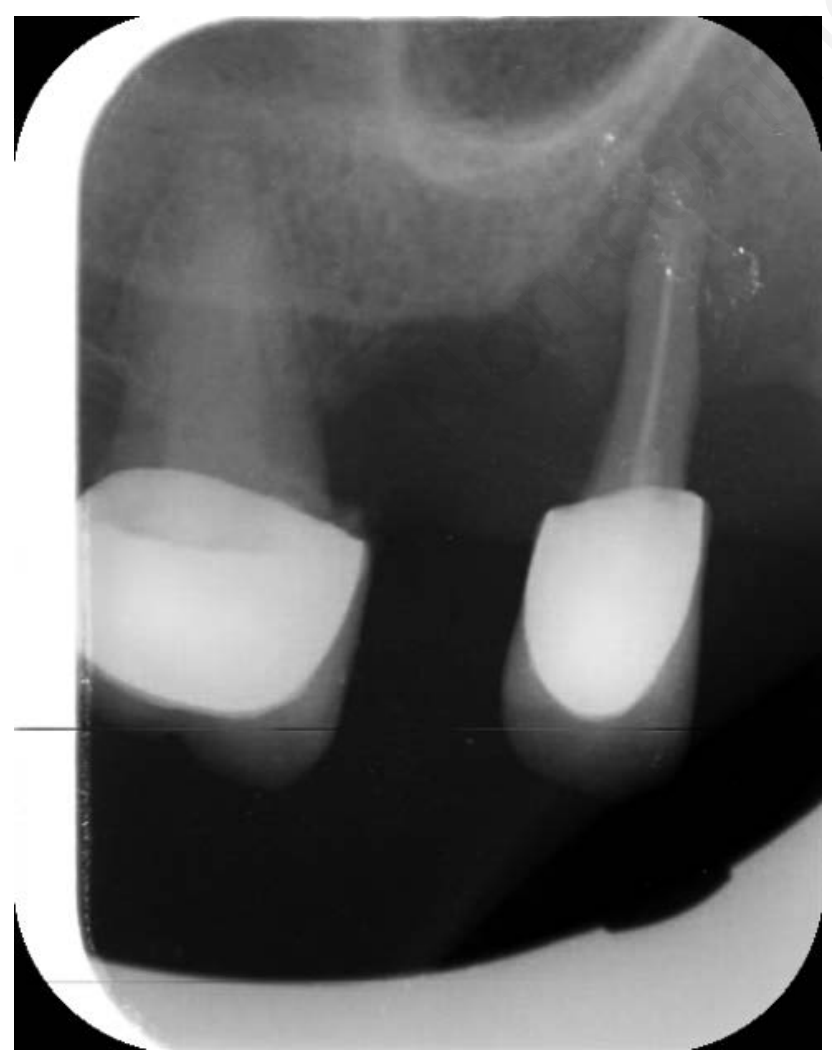

Figure 2. Periapical radiograph shows no osseous involvement. excision of lesion, the patient denied replacement of dental restoration. During minor recurrence in gingival inflammation was noticed, as expected (Figure 6).

\section{Discussion}

Oral PG is a common reactive lesion that can appear at any age, but is frequently seen in patients between the age of 11 and 40. ${ }^{7}$ Women, especially during pregnancy, are more frequently affected than men., Oral PGs have a strong predilection for the gingiva, with up to $70 \%$ of all cases occurring in this site alone. ${ }^{7}$ In the present case, the lesion appeared on the marginal-buccal gingiva of maxillary molar-premolar region.

Clinically, PG is a smooth or lobulated exophytic lesion with small, erythematous papules on a pedunculated or sessile base. It bleeds easily and grows rapidly, and size rarely exceeds $2.5 \mathrm{~cm}$, and is generally asymptomatic and painless. ${ }^{8}$ The surface is

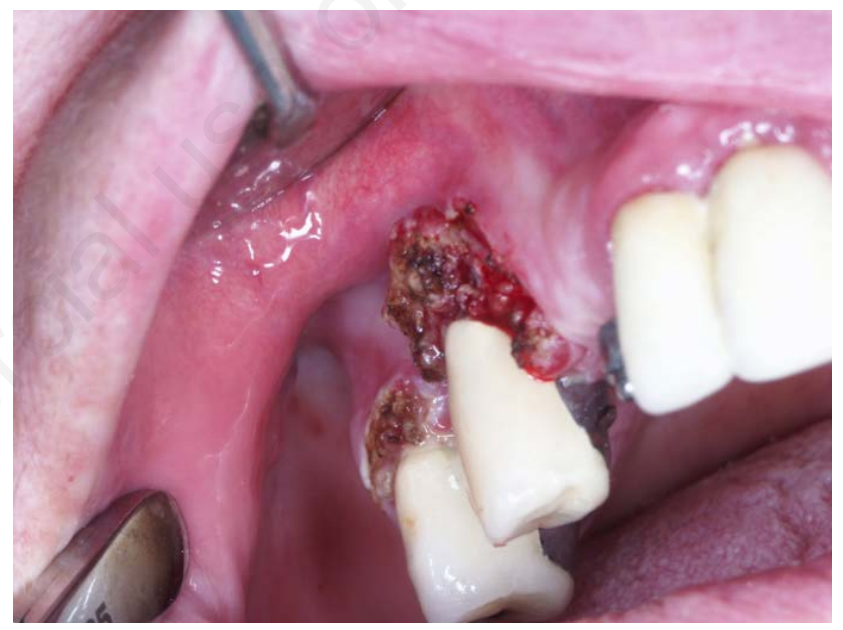

Figure 3. After total excision of the lesion with diode laser.

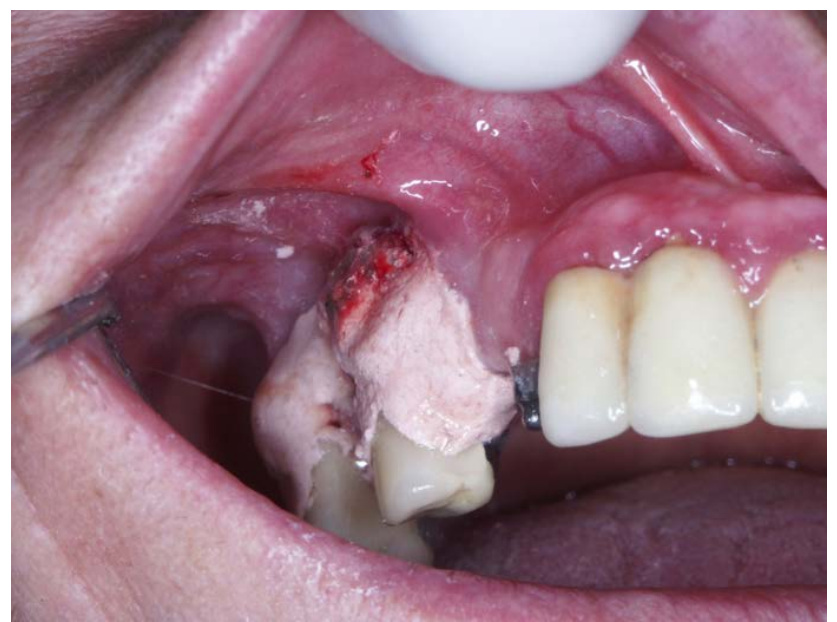

Figure 4. Hyaluronate gel and overlying periodontal dressing was placed and left in situ after excision. 

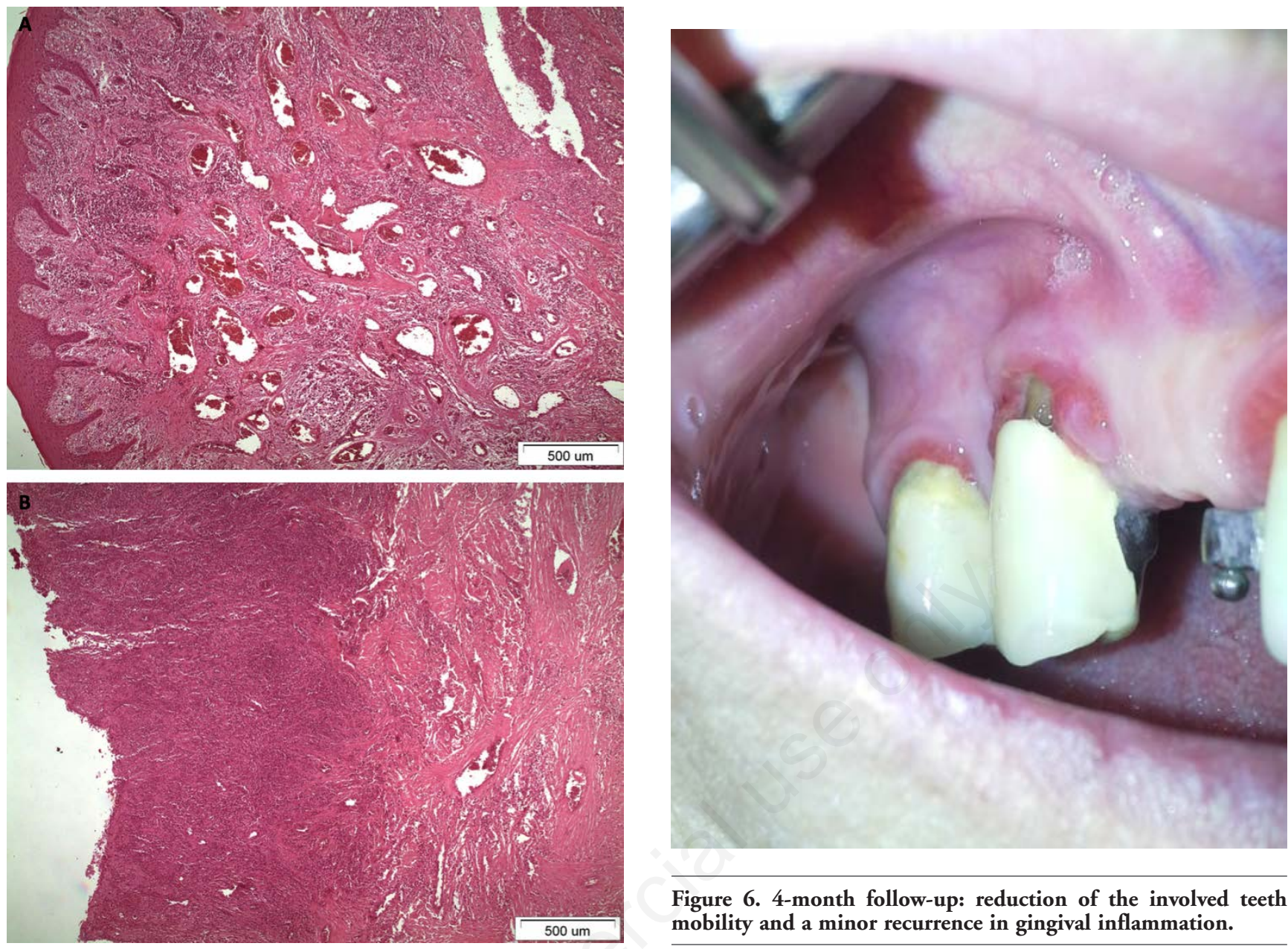

Figure 6. 4-month follow-up: reduction of the involved teeth mobility and a minor recurrence in gingival inflammation.

Figure 5. Histological examination with hematoxylin and eosin staining revealed high vascular proliferation (A) and inflammatory granulomatous tissue (B) with infiltration of lymphocytes, plasma cells and neutrophils and areas of fibrous connective tissue. The lesion was covered by ulcerated stratified squamous epithelium without atypia. These features confirmed the diagnosis of pyogenic granuloma.

often covered by fibrin and is ulcerated and friable due to masticatory trauma. ${ }^{7,8}$ Depending on the age of the lesion, the color of the surface ranges from pink to red or purple. Young PGs have higher vascularity and hyperplastic granulation tissue, while older PGs have more collagen. ${ }^{8}$

Microbial agents such as streptococci and staphylococci may play a role in the etio-pathogenesis of this lesion as they infect minor trauma sites during the healing process and vascular overproduction and tumor-like hyperplasia appears as a response. ${ }^{4,8}$ In the present case, poor oral hygiene (dental calculus) and irregular margins of the metal-ceramic crowns were the major predisposing factors. Differential diagnosis mainly includes vascular tumors like haemangioma, ${ }^{9}$ oral fibroma, ${ }^{10}$ peripheral giant cell granuloma, peripheral ossify- ing fibroma ${ }^{11,12}$ or neoplastic lesions like Kaposi sarcoma, ${ }^{13}$ metastatic carcinoma and other malignant tumors. ${ }^{14,15}$ Drug induced gingival enlargement is sometimes noticed in Nifidepine users. Drug induced gingival enlargement is pale-pink and is a generalized fibrotic event involving a large portion of upper and lower gingiva with a pebbly surface.

In a case report by Kocaman et al. where Nd:YAG laser was used for treatment of PG, bleeding time and operating time were reduced during surgery. ${ }^{16}$ Also rapid postoperative hemostasis was achieved, and no scarring and discomfort were observed. ${ }^{16}$ In a report by Fekrazad et al. who implemented Er:YAG laser for excision of PG, the authors claimed that $\mathrm{CO}_{2}$ and Er:YAG lasers are more suitable means for cutting compared to Nd:YAG and diode lasers, due to their high water absorption, less penetration and coagulation. ${ }^{6}$ Fekrazad et al. also postulated that Er:YAG laser can be considered as an effective and safe technique for excision of PG with minimal invasion and superior clinical advantages such as less intra-operative bleeding, and reduced pain and time of healing. ${ }^{6}$

The diode laser is manufactured from solid semi-conductor crystals made from aluminum $(800 \mathrm{~nm})$ or indium $(900 \mathrm{~nm})$, gallium and arsenic. The coherent laser beam at these particular wavelengths penetrates deep into the mucosa and is highly attenuated by the pigmented tissue, and at the same time is poorly absorbed by the dental hard tissues, so surgery can be safely performed. These lasers can also stimulate fibroblastic proliferation at low energy. ${ }^{5}$ Asnaashari et al. after reporting the removal 
of a pediatric PG using a diode laser, concluded that lasers would reduce stress and fear in pediatric patients and would also minimize discomfort both during and after surgery. ${ }^{17}$ Azma et al. also proposed that diode lasers can be used in oral soft tissue surgery, especially in the context of small prominent lesions because of easy application, better coagulation, no need for suturing, less swelling and pain, and from an esthetic point of view for its potential in the treatment of physiologic gingival pigmentation. It can be considered as a first choice despite periodontal surgery due to; faster action, better re-epithelialization rates, little bleeding and better repair. ${ }^{18}$ Al-Mohaya et al. Implemented diode laser for excision of PG in an uncontrolled diabetic, and patient demonstrated a bloodless surgical site and excellent post-operative healing. ${ }^{3}$ In the current study, diode laser was selected as the treatment of choice because of the excessive size of the lesion $(\sim 2 \times 2 \mathrm{~cm})$, which is significantly larger than many laser operated PG cases in the literature. However, it is critical to correct the margins of closely associated prosthetic restorations, in order to avoid future recurrence.

\section{Conclusions}

It is preferable to excise oral pyogenic granuloma with proper but not extended surgical management for the maintenance of gingival tissue, and from this standpoint the diode laser is a good treatment option. In addition to removal of lesion, the correction or replacement of associated dental restorations is crucial to avoid future recurrence. Excessive bleeding, poor wound healing and recurrent infection are well known in clinical periodontology, and inno- vative approaches like laser surgery can obliterate these biological and operative complications and is thus recommended for routine clinical use.

\section{References}

1. Gex-Collet C, Itin P, Scherer K. Pyogenic granuloma treated with pulsed Nd:YAG (1064 nm) laser- A case series. Med Laser Appl 2008;23:115-9.

2. Hammes S, Kaiser K, Pohl L, et al. Pyogenic granuloma: treatment with the 1,064-nm long-pulsed neodymiumdoped yttrium aluminum garnet laser in 20 patients. Dermatol Surg 2012;38: 918-23.

3. Al-Mohaya MA, Al-Malik AM. Excision of oral pyogenic granuloma in a diabetic patient with $940 \mathrm{~nm}$ diode laser. Saudi Med J 2016;37:1395-400.

4. Rai S, Kaur M, Bhatnagar P. Laser: a powerful tool for treatment of pyogenic granuloma. J Cutan Aesthet Surg 2011;4:144-7.

5. Chaya D, Pankaj G. Lasers in dentistry: a review. Int J Adv Health Sci 2015;2:713.

6. Fekrazad R, Nokhbatolfoghahaei H, Khoei F, Kalhori K. Pyogenic granuloma: surgical treatment with Er:YAG laser. J Lasers Med Sci 2014;5:199-205.

7. Reichart P, Philipsen HP. Color atlas of dental medicine, oral pathology. 1st ed. USA: Thieme; 2000. pp.163-164.

8. Jafarzadeh H, Sanatkhani M, Mohtasham N. Oral pyogenic granuloma: a review. J Oral Sci 2006;48:16775.

9. Patil A, Pattanshetti C, Varekar A, Huddar SB. Oral capillary haemangioma mimicking pyogenic granuloma: a challenge for diagnosis and management. BMJ Case Rep 2013;2013:pii:bcr2012007874.

10. Krishnan V, Shunmugavelu K. A clinical challenging situation of intra oral fibroma mimicking pyogenic granuloma. Pan Afr Med J 2015;22:263.

11. Sridhar R, Wanjari S, Kanteshwari IK. Interrelationship between pyogenic granuloma and peripheral ossifying fibroma: a case report. J Dent Hyg 2012;86:179-84.

12. Prasad S, Reddy SB, Patil SR, et al. Peripheral ossifying fibroma and pyogenic granuloma. Are they interrelated? N Y State Dent J 2008;74:50-2.

13. Scully C. Oral and Maxillofacial Medicine. The Basis of Diagnosis and Treatment. 2nd ed. London: Elsevier; 2008. pp. 367.

14. Ababneh K, Al-Khateeb T. Aggressive pregnancy tumor mimicking a malignant neoplasm: a case report. J Contemp Dent Pract 2009;10:E072-8.

15. Ramirez RJ, Seoane J, Montero J, et al. Isolated gingival metastasis from hepatocellular carcinoma mimicking a pyogenic granuloma. J Clin Periodontol 2003;30:926-9.

16. Kocaman G, Belduz N, Erdogan C, et al. The use of surgical Nd:YAG laser in an oral pyogenic granuloma: A case report. J Cosmet Laser Ther 2014;16:197-200.

17. Asnaashari M, Mehdipour M, MoradiAbbasabadi F, Azari-Marhabi S. Expedited removal of pyogenic granuloma by diode laser in a pediatric patient. J Lasers Med Sci 2015;6:40-4.

18. Azma E, Safavi N. Diode laser application in soft tissue oral surgery. J Lasers Med Sci 2013;4:206-11. 